\title{
Age-related histological changes in rat tibia
}

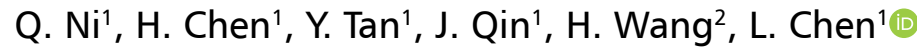 \\ 'Department of Orthopaedic Surgery, Zhongnan Hospital of Wuhan University, Hubei Provincial Key Laboratory \\ of Developmentally Originated Disease, Wuhan, China \\ 2Department of Pharmacology, Basic Medical School of Wuhan University, Hubei Provincial Key Laboratory \\ of Developmentally Originated Disease, Wuhan, China
}

[Received: 19 August 2020; Accepted: 27 October 2020; Early publication date: 3 November 2020]

Background: At present, studies on osteochondral morphogenesis only focus on a certain period of time or only provide a pattern diagram, but lack of dynamic tracking observation from the initiation of development to maturity. This study was aimed to dynamically observe the changes of skeleton morphology and structure from embryo to adult, to provide research data for enriching the knowledge of bone and cartilage tissue structure. Materials and methods: In the intrauterine experiment, 5 normal pregnant Wistar rats were sacrificed under anaesthesia at gestational day (GD) 14,17, 20, respectively. One of their offspring was randomly selected, and a total of 5 offspring were obtained at each time point. In the postnatal experiment, on the $7^{\text {th }}$ and $10^{\text {th }}$ day after birth and at postnatal weeks (PW) 2, 3, 6, 12, 28, 5 offspring rats from 5 different pregnant Wistar rats were randomly selected and sacrificed under anaesthesia at each time point. After obtaining the above offspring, the soft tissue was removed, and the tibia of hind limbs was retained for paraffin-embedded section. After stained with Safranin-O-fast-green and haematoxylin, the morphological development of the tibia was observed under an optical microscope. Results: At GD14, there was no obvious joint space, the whole hind limb was cartilage and bone tissue was not visible. At GD17, visible joint space was seen and the chondrocytes in the centre region appeared to hypertrophy. At GD20, the primary ossification centre was obvious, and a typical epiphysis growth plate structure could be seen. On the $7^{\text {th }}$ day after birth, the chondrocytes in the centre of epiphysis cartilage were hypertrophic and differentiated, the cartilage canal grows from the cartilage surface toward the centre of the epiphysis cartilage, at postnatal day 14, the secondary ossification centre was formed. At this time, the tibia had typical morphological characteristics of the metaphysis, however, there was no obvious layered structure of articular cartilage; the stratified structure of articular cartilage could be seen at PW6, but its mature marker (tidemark) was still not visible; however, at PW12, typical 4 layers of articular cartilage appeared, and the tidemark was visible. The growth plates were clearly visible at PW2, 6 and 12. At PW28, growth plates could still be observed, but its morphology is abnormal. Conclusions: Our results, for the first time, dynamically observed the morphological changes of osteochondral at critical period of development from embryo to adult, especially the process of cartilage canal participating in the formation of secondary ossification centre. (Folia Morphol 2021; 80, 4: 1005-1019)

Key words: osteochondroplasia, cartilage anlage, primary ossification centre, secondary ossification centre, cartilage canal

Address for correspondence: Dr. L.B. Chen, Department of Orthopaedic Surgery, Zhongnan Hospital of Wuhan University, Wuhan 430071, China, tel: +86-13618610516, fax: +86 2767812892, e-mail: Ibchen@whu.edu.cn; Dr. H. Wang, Department of Pharmacology, Basic Medical School of Wuhan University, Wuhan 430071, China, tel: +86-13627232557, fax: +86 2768758665, e-mail: wanghui19@whu.edu.cn

This article is available in open access under Creative Common Attribution-Non-Commercial-No Derivatives 4.0 International (CC BY-NC-ND 4.0) license, allowing to download articles and share them with others as long as they credit the authors and the publisher, but without permission to change them in any way or use them commercially. 


\section{INTRODUCTION}

Bone and cartilage are important structures which form the framework of the body, supporting, protecting and moving the body. Bones in different parts of the skeleton develop through two distinct processes [10], intramembranous ossification and intracartilaginous ossification. In intramebranous ossification, bones ossify directly without passing from the connective tissue structure to the cartilage tissue. In the intracartilaginous ossification type, which occurs in the axial and appendicular skeleton of vertebrates, the bones first turn into hyaline cartilage tissue and then into bone tissue [26]. Intracartilaginous ossification is initiated during foetal life, and continues until growth ceases in early adulthood. Limb development begins as a mesenchymal cell proliferation from the lateral plate mesoderm. These cells give rise to a circular bulge, known as the limb bud. Mesenchymal cells in the limb bud first undergo cellular condensation. This condensation results in a dramatically increased cell density that is a prerequisite for chondrogenesis. Following condensation, cells at the centre begin to differentiate into the chondrogenic lineage and turn the mesenchymal condensation into a primordial cartilage anlage. The cartilage anlage represents the moulds of the future skeletal elements; the entire model of the bone is still in a cartilage phase. When mesenchymal condensations form, several layers of mesenchymal cells at the boundary become fibroblast-like cells and eventually establish the perichondrium [30]. Shortly after the initiation of the cartilage anlage, chondrocytes at the centre of individual cartilage anlages undergo hypertrophy. These pre- and early hypertrophic chondrocytes in the cartilage anlage secret Indian hedgehog protein to directly stimulate differentiation of their surrounding perichondrial cells into osteoblast lineage cells [23], including osteoprogenitors and osteoblasts, these secrete a characteristic matrix, forming a bone collar [20]. The bone collar surrounding the central region of cartilage anlage and eventually establishes the midshaft (diaphyseal) cortical bone. Blood vessels, osteoclasts, as well as bone marrow and osteoblast precursors then invade the model from the bone collar and proceed to form the primary ossification centre (POC) [36]. In long bones, the secondary ossification centre (SOC) subsequently forms shortly after birth at each end of the cartilage anlage, leaving a cartilaginous growth plate between the POC and SOC, as well as the prospective permanent articular cartilages at each end of the bone [26]. The growth plate is responsible for longitudinal growth of bones. Skeletal maturity occurs when the expanding POC meets the SOC, thus obliterating the growth plate [9].

As an important tissue type in vertebrates, chondrocytes are involved in the whole process of the genesis and development of bone and cartilage system [30], so it is of great significance to study the biological mechanism of bone growth and development to explore the morphological characteristics and rules of chondrocyte differentiation in the process of osteochondrogenesis, and to uncover the pathogenesis of skeletal system diseases. However, there are few studies on characteristics of chondrocyte changes during chondrogenic formation, differentiation and intracartilaginous ossification.

In bone research, rat is the most important model system at present, rat is more similar to humans in their philicity and their skeletal phylogenetic processes are more similar, so it remains the main animal model $[2,4]$. Therefore, the purposes of the present study were to determine the sequential change of histologic events involved in the formation of long bones and their epiphysis from the embryonic limbbud stage to skeletal maturity, to classify the various stages, and to define the time at which each event occurs in the rat. This study can provide research data for enriching the knowledge of bone and cartilage tissue structure. It also has positive significance for the healthy development of bone during the development period.

\section{MATERIALS AND METHODS}

\section{Chemicals and reagents}

Fast green (catalogue no. 2353-45-9; Sigma-Aldrich, St. Louis, MO, USA) and Safranin-O (catalogue no. Cl-50240; Junsei Chemical Co. Tokyo, Japan); haematoxylin-eosin (H\&E; Sigma).

\section{Animals and treatment}

Specific pathogen free Wistar rats (female: 180 -220 g, male: $260-300 \mathrm{~g}$ ) were obtained from the Experimental Centre of Hubei Medical Scientific Academy (No. 2010-2012, Hubei, China). This experiment was performed in the Centre for Animal Experimentation of Wuhan University (Wuhan, China), which has been accredited by the Association for Assessment and Accreditation of Laboratory Animal Care International (AAALAC International). All animal experimental procedures were approved by the Chinese Animal Welfare 
Committee and performed in accordance with their Guidelines for the Care and Use of Laboratory Animals.

Animals were housed (room temperature: $18-22^{\circ} \mathrm{C}$; humidity: 40-60\%), acclimated, and mated. Upon confirmation of mating by the appearance of sperm in a vaginal smear, the day was taken as gestational day (GD) 0. Pregnant rats were transferred to individual cages.

In the intrauterine experiment, 5 normal pregnant Wistar rats with 10-14 live foetuses were anesthetized with isoflurane and euthanized on GD 14, 17, 20 , respectively. One of their offspring was randomly selected, and a total of 5 offspring were obtained at each time point. For experiments on postnatal rats, 5 pregnant rats were kept until normal delivery on GD21. On postnatal day 1 (PD1), the numbers of pups were normalised to 8 pups per litter to assure adequate and standardised nutrition until weaning (postnatal week 4, PW4). On the $7^{\text {th }}$ and $10^{\text {th }}$ day after birth and at PW 2, 3, 6, 12, 28, 5 offspring rats from 5 different pregnant Wistar rats were randomly selected and sacrificed under anaesthesia at each time point. After obtaining the above offspring, the soft tissue was removed, and the tibias of hind limbs were retained and fixed in $4 \%$ paraformaldehyde solution for $24 \mathrm{~h}$ before being decalcified in EDTA, dehydrated in alcohol and embedded in paraffin until further histological examination.

\section{Histological assays}

Foetal rat tibia specimens were fixed in $4 \%$ paraformaldehyde at $\mathrm{pH} 7.4$ for 3 days and then processed for paraffin embedding. Adult knee joints were fixed in $4 \%$ paraformaldehyde, decalcified in a $20 \%$ ethylenediamine tetraacetic acid solution for 21 days, and then embedded in paraffin wax. After each paraffin wax was cut to the bone tissue layer, 5 layers were removed and the subsequent layers were retained for staining. The tibias were sectioned sagittally at a thickness of $5 \mu \mathrm{m}$. Safranin-O-fast green staining and H\&E staining were performed by standard procedures [27]. The photo images of histology were captured using the Nikon NIS Elements BR light microscope (Nikon, Tokyo, Japan).

\section{RESULTS}

\section{Dynamic development of osteochondral from intrauterine to postnatal}

As shown in Figure 1, at GD14, the mesenchymal cells aggregate to form a limb bud, which was a car- tilage anlage. There was no joint space at both ends of the cartilage anlages, the chondrocytes homing in the centre of cartilage anlages where the POC is about to form. At GD17, the homing chondrocytes in the centre of cartilage anlages differentiated into hypertrophic chondrocytes, the joint space was clearly visible, but the morphological difference between articular cartilage and epiphysis cartilage was not obvious. At GD20, the POC expanded and the typical epiphysis growth plate cartilage structure was formed at both ends. 10-14 days after birth, the chondrocytes in the central area of the epiphysis cartilage at both ends of the cartilage anlages were hypertrophic and apoptotic, several cartilage canals elongated from different areas of epiphysis cartilage toward the location where the SOC would appear. At PW2, the SOC could be clearly identified. At PW3, the cartilage canals disappeared, forming a typical bone shape during development. At PW6, the articular cartilage had not formed the standard 4-layer structure at maturity, and the tidemark and subchondral bone were not visible. At PW12, articular cartilage was mature with a typical 4-layer structure, the tidemark and subchondral bone could be clearly identified. As the growth plate of rats existed for life, the residual growth plate could still be seen at PW28.

\section{Morphological overview of cartilage anlages and primary ossification centre}

GD14, the cartilage anlages formed by the aggregation of mesenchymal cells was clearly visible, all of it was cartilage, bone tissue was not visible, and all the stains were Safranin-O-fast-green without staining. At this time, there was no obvious joint space, the position where the joint will form was still the dense mesenchymal cells, undifferentiated chondrocytes were the main cells in the cartilage anlages. The chondrocytes in the centre of the cartilage anlages became bigger and formed clusters, the intercellular space was larger than that at both ends, and begin to differentiate into prehypertrophic chondrocytes.

GD17, the joint space at both ends of the cartilage anlages was clearly visible and the anatomical structure of the tibia was clearly defined, the whole tibia was still cartilage, and bone tissue was not visible, all of it was stained with Safranin-O. However, at this time, the volume of the middle segment cells of cartilage anlages increased, showing the typical morphology of hypertrophic chondrocytes, indicating that the 


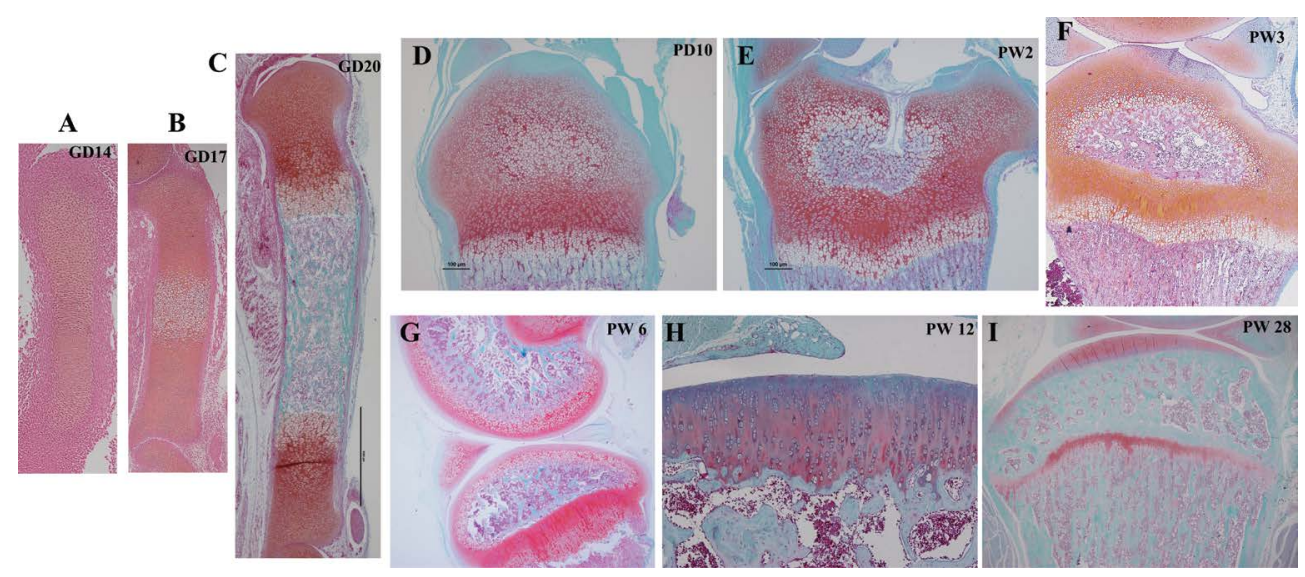

Figure 1. Safranine and fast green double dyeing demonstrates the dynamic development process of osteochondral from intrauterine to postnatal; A. Tibia of offspring rats at gestational day (GD) 14, cartilage anlages is present; B. Tibia of offspring rats at GD17, primary ossification centres appear; C. Tibia of offspring rats at GD20; D. Development of epiphysis at postnatal weeks (PW) 10; E. Development of secondary ossification centre formation at PW2; F. Development of secondary ossification centre formation at PW3; G. Development of articular cartilage at PW6; H. Development of articular cartilage at PW12; I. The whole osteochondral was observed at PW28.
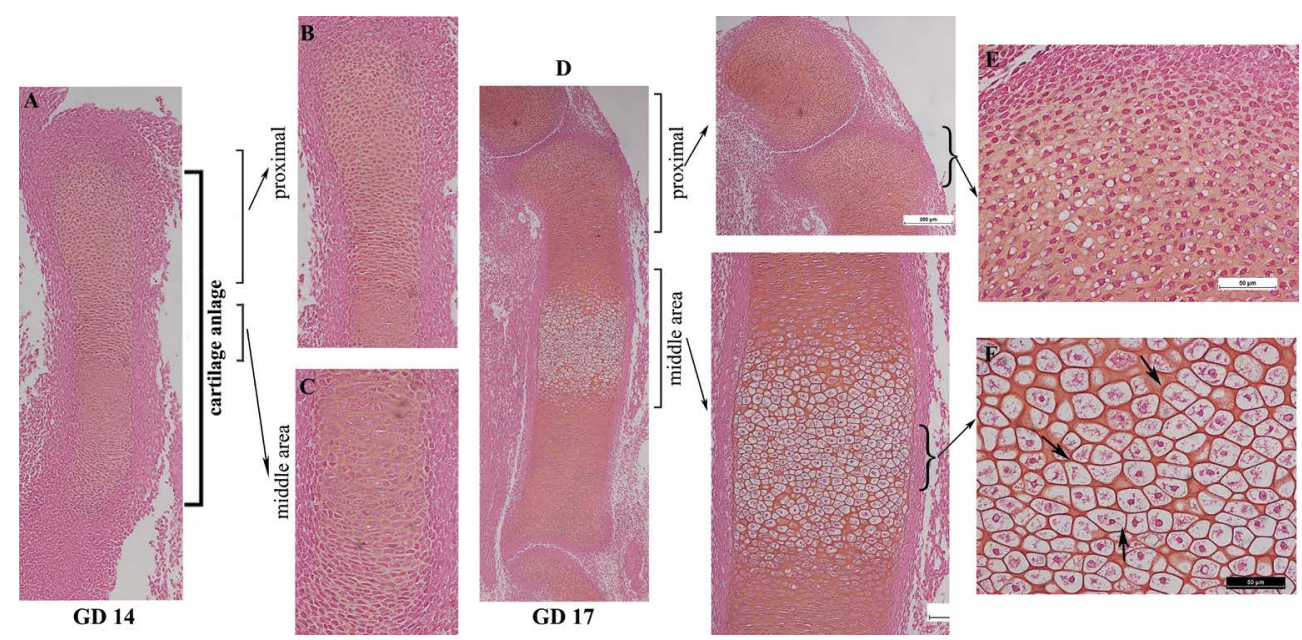

GD 17

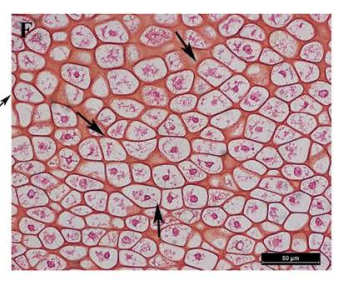

Figure 2. Morphology of the tibial cartilage anlage at gestational day (GD) 14, 17; A. Gross morphology of cartilage anlage at GD14; B. Development of cartilage anlage in the proximal at GD14; C. Development of the middle segment of cartilage anlage at GD14; D. Gross morphology of cartilage anlage at GD17; E. Development of cartilage primordium in the proximal at GD17; F. Development of the middle segment of cartilage anlage at GD17 (the black arrow refers to hypertrophic chondrocytes).

POC was about to be formed. With the hypertrophy of chondrocytes in the central position, the cartilage anlages began to stratify, the cells close to the joint surface were densely arranged and uniform in shape and size, with the characteristics of stem cells. From the joint to the centre, the cell volume gradually increased and hypertrophied. The results showed that the cartilage anlage was a model of the future skeletal elements, and the chondrocytes in the middle of the cartilage anlage developed hypertrophy before the cartilage anlage ossified. When the cartilage anlage was ossified, the chondrocytes in the middle were the first to become hypertrophy (Fig. 2D-F).

\section{Osteochondral development on the day before birth}

GD20, which is the day before birth, the bone further developed and matured, the longitudinal length of the tibia became longer and the transverse width increased, the two ends were still cartilage and stained with Safranin-O. At this time, a mature POC with fastgreen coloured bone tissue and typical medullary cavity structures could be observed. A typical epiphysis growth plate structure located at both ends of tibia could be seen. From the surface to the deep layer of cartilage, chondrocytes gradually matured; cells varied in morphology, with distinct cell layers, more regular, 


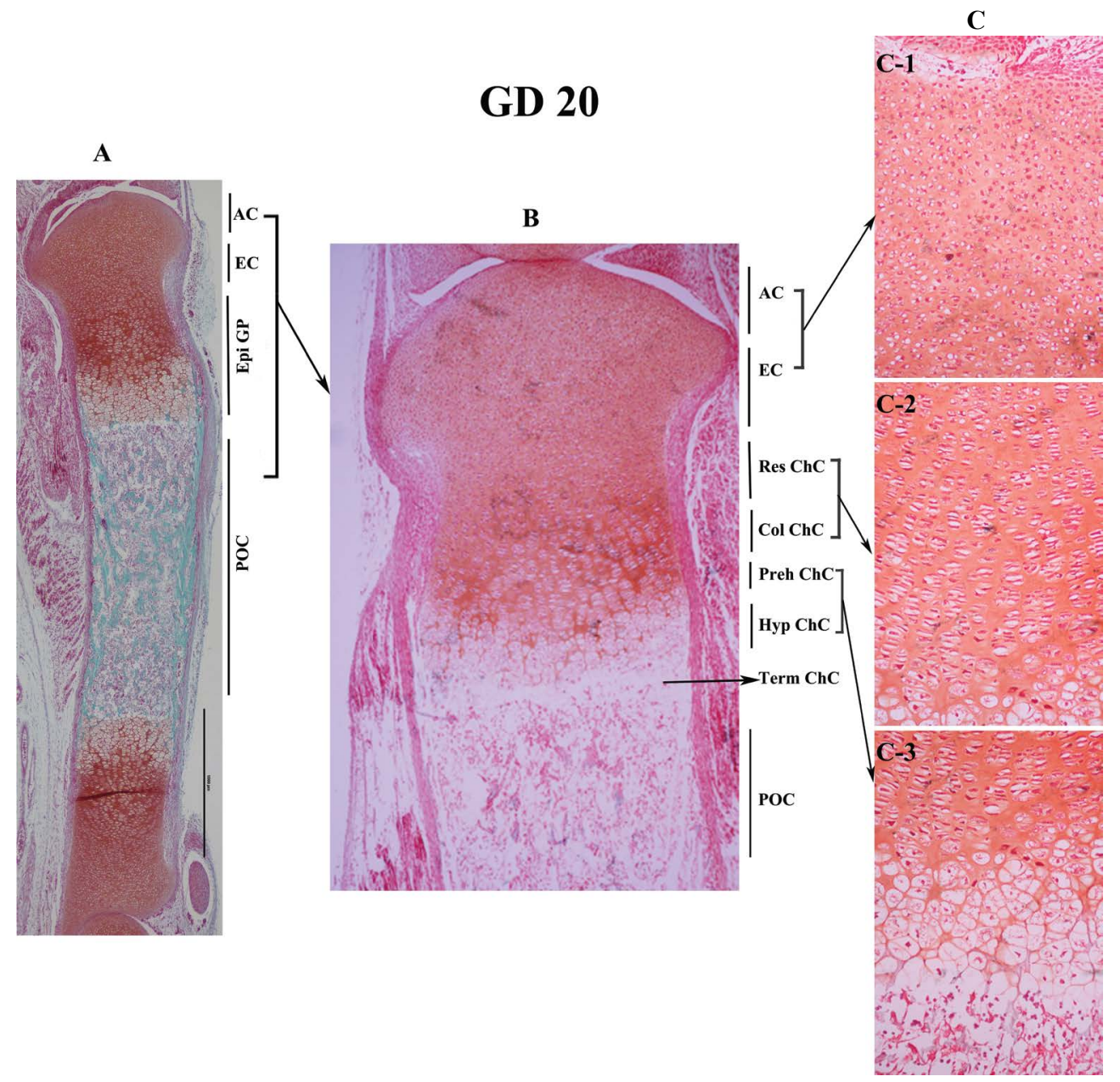

Figure 3. Morphological structure of the tibia and epiphysis at gestational day (GD) 20 (the day before birth); A. In general view of the whole tibia, the primary ossification centre in the centre and epiphysis growth plates at both ends were clearly visible; B. General view of epiphysis growth plate adjacent to knee joint; $\mathbf{C}$. General view of cells in different regions of epiphysis; AC — articular cartilage; EC — epiphysis cartilage; Epi GP — epiphysis growth plate; POC — primary ossification centre; Res $\mathrm{ChB}$ - resting zone chondroblast; Col ChB — columnar chondroblast; Preh $\mathrm{ChC}$ - prehypertrophic chondrocyte; Hyp ChC — hypertrophic chondrocyte; Term ChC — terminal chondrocyte (chondro-osseous junction).

orderly arrangement, and regular distribution. It could be roughly divided into articular cartilage, epiphysis cartilage, epiphysis growth plate, terminal chondrocyte (chondro-osseous junction) and POC (Fig. 3B). In addition, the epiphysis growth plate could be further subdivided into resting zone, proliferating zone, prehypertrophy and hypertrophy zone (Fig. 3B). In the cartilaginous reserve region, chondrocytes were elliptic, with a large number and dense arrangement, the arrangement direction was parallel to the articular surface, and still retains the characteristics of stem cells. The chondrocytes in the proliferating zone were proliferated by the reserved chondrocytes and arranged in series. The cell volume increased, the cells appeared in pairs and groups, and the intercellular space increased. Subsequently, the hypertrophic chondrocytes became round and enter the morphology of the pre-hyper- trophy chondrocytes, which were further differentiated into hypertrophic chondrocytes. The hypertrophic chondrocytes were close to the $\mathrm{POC}$ and have the largest volume (Fig. 3C). Each cell existed independently, and eventually apoptosis and osteogenesis. At the same time, there was a layer of terminal chondrocyte (chondro-osseous junction) in the interface between hypertrophy chondrocytes and osteoblasts.

\section{Formation and morphological characteristics of secondary ossifying centres}

In rats, SOC is often found in PW2, but the formation of $\mathrm{SOC}$ is a dynamic process, which may start from PW1. Similar to the formation of POC, partial chondrocyte that in the region where the ossification centre is about to appear are hypertrophic and apoptotic, but what is different is that the formation 


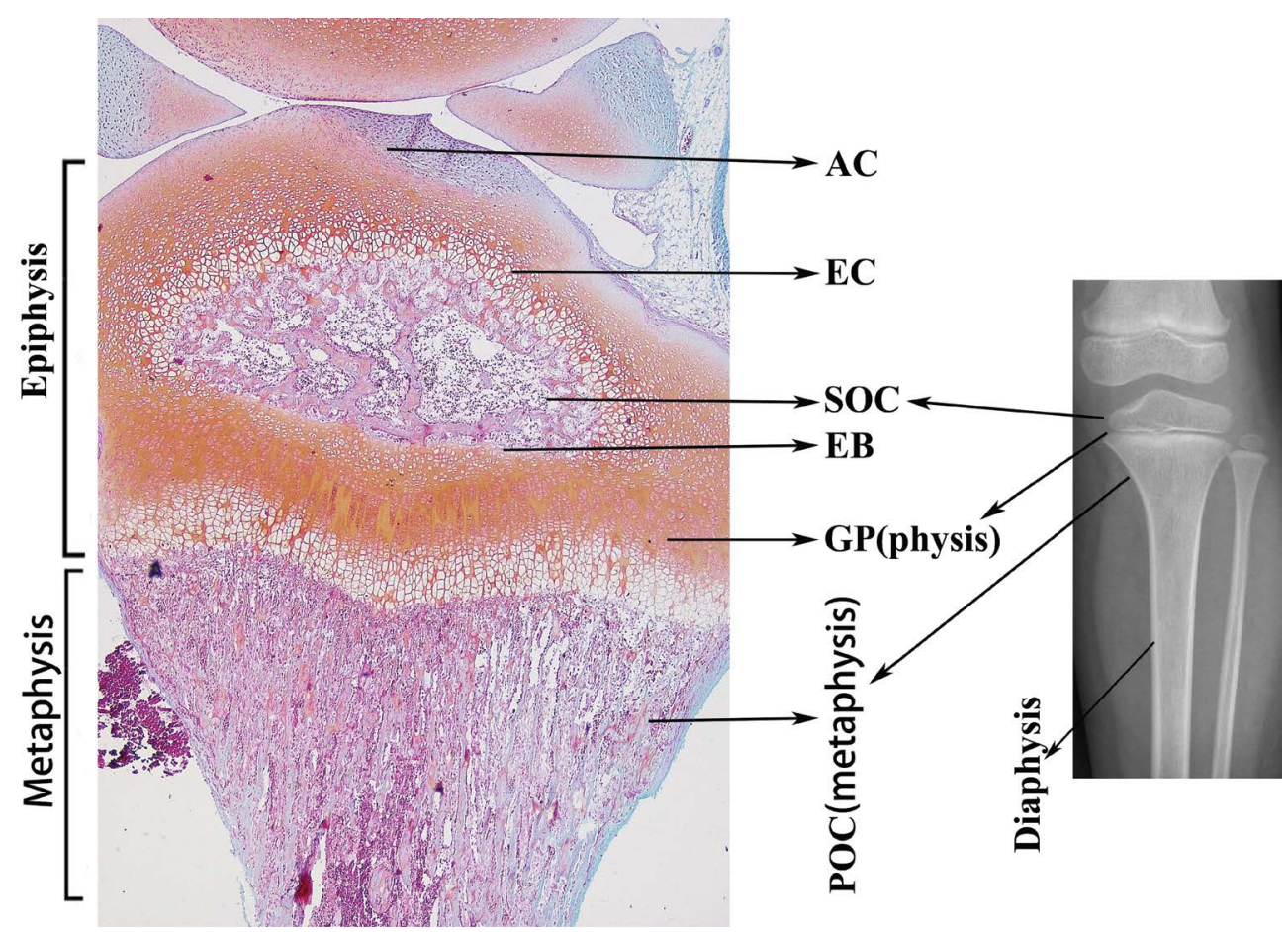

Figure 4. The histologic structure of the epiphysis of the proximal tibia is illustrated. The entire developing end of the bone from the articular cartilage surface to the last cells of the hypertrophic zone of the growth plate is the epiphysis. This encompasses three regions that are initially cartilage: (a) the articular cartilage (AC), (b) the growth plate (GP), also referred to as the epiphysis growth plate or the physis and (c) the epiphysis cartilage $(\mathrm{EC})$ which refers to the cartilage mass between the articular cartilage and the growth plate cartilage. The secondary ossification centre (SOC) forms by the endochondral mechanism within the epiphysis cartilage. The epiphysis border (EB) is the boundary between the growth plate and the SOC; POC — primary ossification centre.

of SOC involves the participation of cartilage canal. In the early stage of SOC formation, several vascular-like cartilage canals elongate from different areas of epiphysis cartilage toward the location where the SOC will appear. These cartilage canals communicate with the tissue with abundant blood supply on the surface of cartilage, and allow for the arrival of blood vessels, vasoactive substances and osteoprogenitors to the centre of the epiphysis cartilage to promote the SOC formation. However, the cartilage canal was no longer visible at PW3 (Fig. 4). Once the SOC is formed, there were more than three ossification centres in the long bone, one in the centre of the diaphysis and the other at both ends. At this stage, the typical bone morphology of the development period was formed, that was, from the joint space to the centre of the diaphysis, in the order of articular cartilage, epiphysis cartilage, SOC, growth plate and POC (Fig. 4).

Cartilage canal, as shown in Figure 5A, the formation of cartilage canal generally began at multiple points simultaneously, the initial structure of the cartilage canal was similar to the structure of the ossification centre, and gradually expanded to form a tubular structure. It joined the SOC with the tissue with abundant blood vessels on the surface of cartilage. Cartilage canal is a tubular structure surrounded by loose connective tissue, with a diameter of $50-150 \mu \mathrm{m}$; the cartilaginous wall is continuous with the pericardium, and there are red blood cells in the lumen, it's ends like a capillary bulb. When the SOC was mature, the cartilage canal which passed through the non-vascular tissue was closed again, so as to maintain the vascularization of cartilage in the proximal joint area (Fig. 1F).

Articular cartilage, at PW2, a large SOC could be seen in the centre of epiphysis, the cartilage between the ossification centre and the cartilaginous surface was articular cartilage (Fig. 5C, D). The volume of chondrocytes was larger than that of GD20, and the intercellular space increased. However, it was still difficult to distinguish articular cartilage from the underlying epiphysis cartilage by histology. They could be roughly divided into 3 layers: the chondrocytes in the surface layer were flat and densely arranged, the volume of chondrocytes in the middle layer increased and show the oval-shape, 
A Formation of SOC and dynamic change of $\mathrm{CC}$
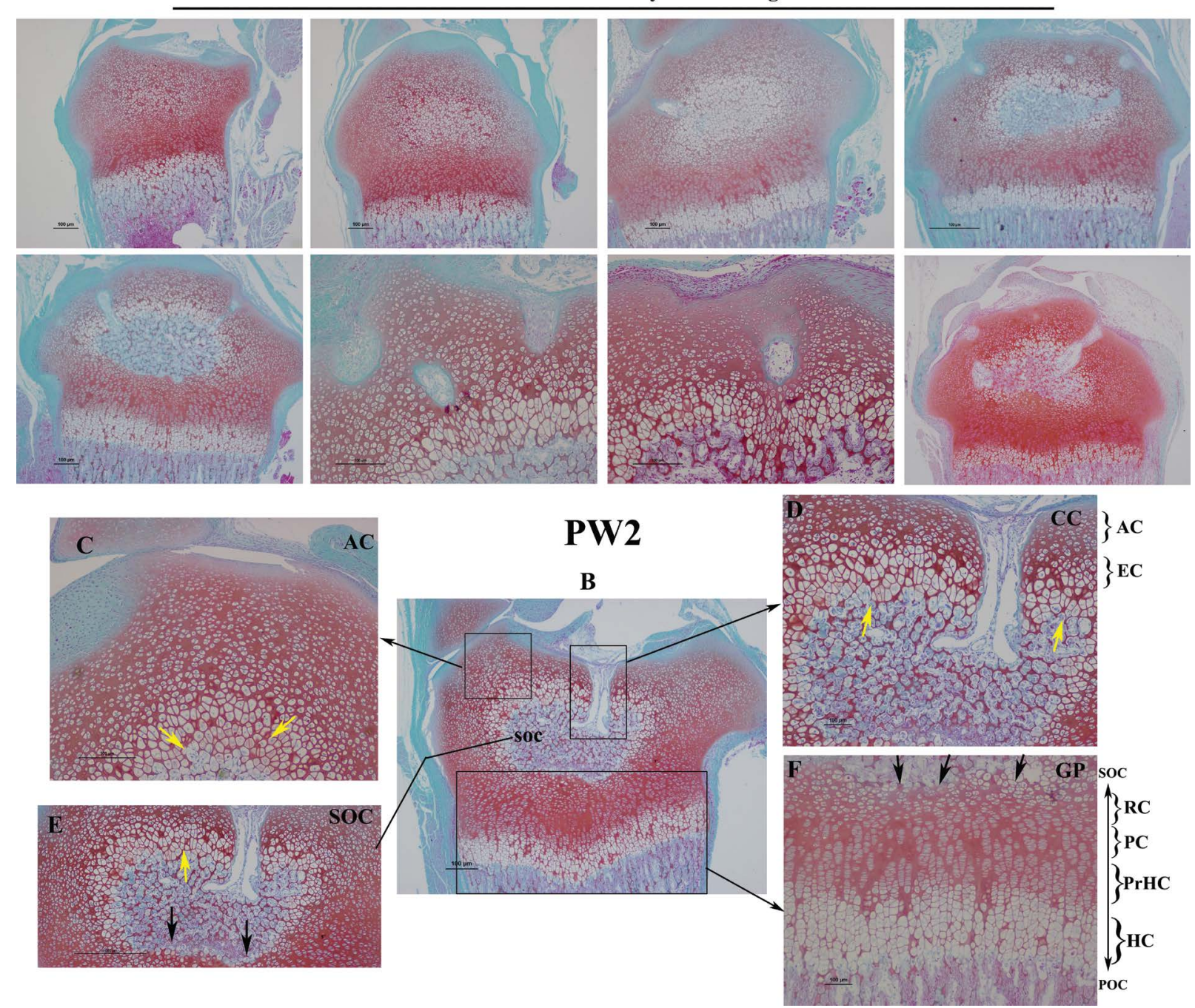

Figure 5. The dynamic process of secondary ossification centre (SOC) formation and its characteristics; A. The dynamic process of SOC formation and the dynamic change of cartilage canal (CC) at different developmental time; B. Gross morphology of epiphysis at postnatal weeks (PW) 2; C. Gross morphology of articular cartilage (AC) at PW2; D. Gross morphology of cartilage canals at PW2; E. Gross morphology of SOC at PW2 (the yellow arrow shows the hypertrophic chondrocytes near the articular side, and the black arrow indicates the border of the epiphysis border near the growth plate side); $\mathbf{F}$. Gross morphology of growth plate cartilage at PW2 (the black arrow indicates the border of the bone plate near the growth plate side); $\mathrm{RC}$ — resting cartilage; PC — proliferative cells; $\mathrm{PrHC}$ — prehypertrophic chondrocyte; $\mathrm{HC}$ hypertrophic chondrocyte; POC — primary ossification centre.

and agglomerated into clusters, the deep layer was adjacent to the SOC, and the chondrocytes were hypertrophic (Fig. 5C). Vascular invasion could be seen at the edge of articular cartilage, and cartilage canal could be seen in the centre of articular cartilage, and the cells around the cartilage canal were hypertrophic chondrocytes.

Secondary ossification centre, from $7^{\text {th }}$ day after birth, chondrocytes at the centre of the epiphysis cartilage underwent hypertrophy and matrix mineralisation, the cartilage canals extended to this region from different positions, and finally the inner ends of the canal fused and expanded by continued excavation of surrounding hypertrophic chondrocytes to create the space for bone and bone marrow, thus establishing the SOC. After the formation of ossification centre, with the invasion of a large number of blood vessels, the SOC began a centripetal and continuous process of chondrocyte proliferation, hypertrophy, transformation, apoptosis, vascular invasion and other new bone formation processes from the inside to the outside. The SOC had a process of gradual ossification and expansion. When the $\mathrm{SOC}$ expanded close to the epiphysis plate, its shape changed from spherical to 


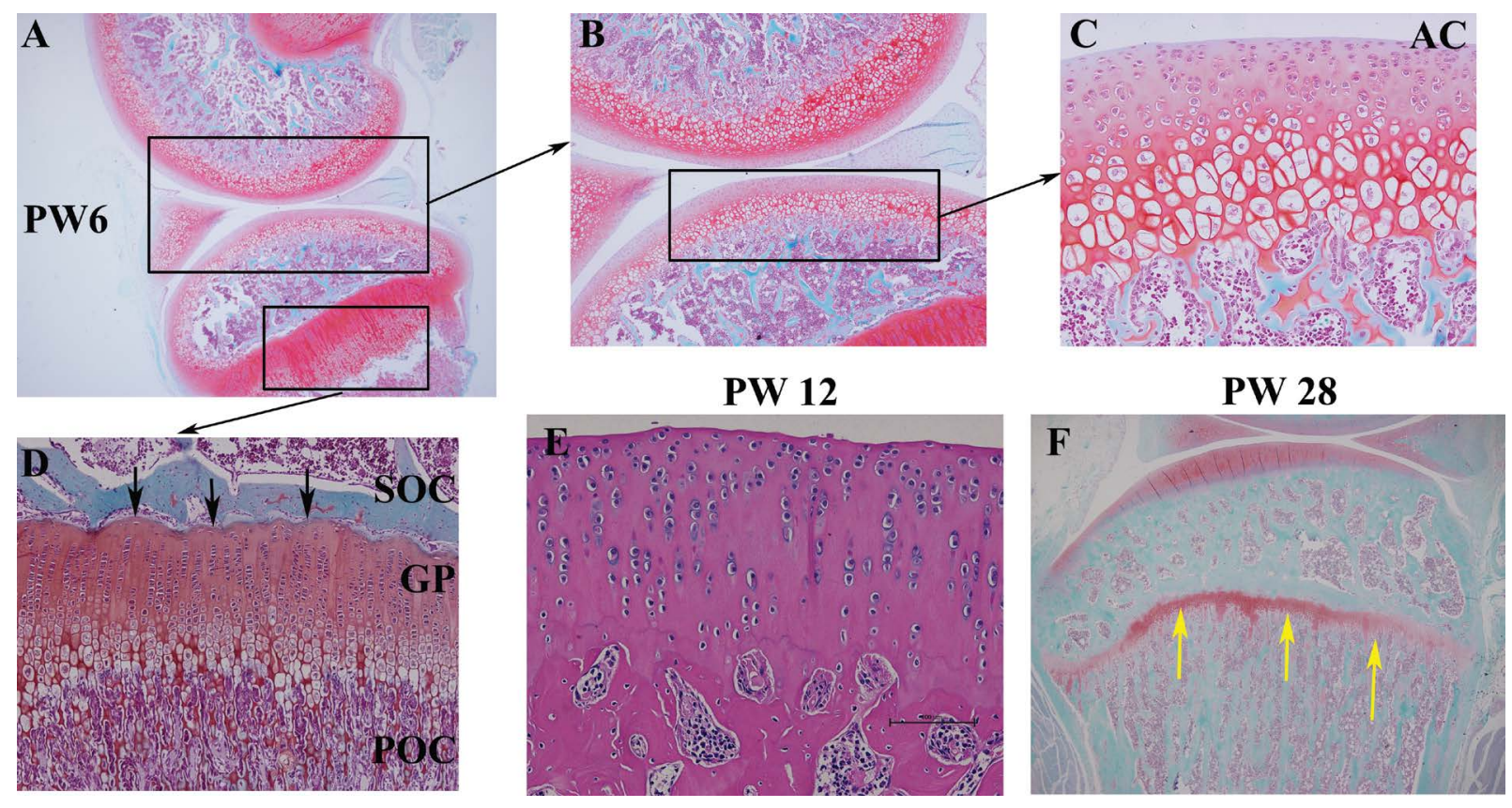

Figure 6. Morphological characteristics of mature osteochondral; A. The gross morphology of metaphysis at postnatal weeks (PW) 6; B. Gross morphology of articular cavity and articular cartilage during at PW6; C. General morphology of articular cartilage (AC) at PW6; D. General morphological view of the growth plate (GP) at PW6 (the black arrow indicates the boundary of the epiphysis border near the side of the growth plate); SOC — secondary ossification centre; POC — primary ossification centre; E. Gross morphology of articular cartilage at PW12; F. General morphological view of metaphysis at PW28 (the yellow arrow refers to the degenerated growth plate).

flat, and its longitudinal axis gradually parallel to the metaphyseal. Morphological observation showed that the osteogenesis of the SOC adjacent to the articular space was different from that of the growth plate, chondrocytes directly differentiate into hypertrophic chondrocytes from resting chondrocytes, there was no longitudinal proliferation and division process. In the above process, chondrocytes did not experience the obvious proliferation stage and pre-hypertrophy stage. Compared with the process of epiphysis ossification, we found that chondrocytes in epiphysis cartilage did not undergo columnar chondrocyte proliferation. However, no obvious hypertrophic chondrocytes could be observed in the side of the SOC near the growth plate, and there was a well-defined osseous boundary, which was called epiphysis border, which might be related to the prevention of ossification of the reserved cartilage layer of the growth plate (Fig. 5E).

Growth plate, as shown in Figure 5F, at PW2, the growth plate had a clearer 4 discrete zones, and its morphology was consistent with that of the epiphysis growth plate at GD20, which was located between the SOC and the POC.

\section{Morphological characteristics of mature osteochondral}

As shown in Figure 6, at PW6, the structure of the joint (meniscus, cruciate ligament) was clearly visible, and the joint structure was mature. The ossification centre continues to expand, and there was no cartilage canal in the articular cartilage. However, the articular cartilage was still not fully developed at this time, the 4-layer structure of the articular cartilage was still unclear, and the cell arrangement had no obvious characteristics, the area near the SOC was still hypertrophic chondrocytes (Fig. 6C). Calcified cartilage layer, tidemark, subchondral bone was still not recognised (Fig. 6C). At this time, the growth plate was still clearly visible, and its shape, length and width were not significantly different from those of PW2, but the epiphysis border that is the boundary between the growth plate and the SOC, was clearer. At PW12, H\&E staining showed that articular cartilage was mature, and its 4-layer structure was clearly visible. In addition, the tidemark, a marker of mature articular cartilage, was clearly visible, and the subchondral bone could be clearly identified (Fig. 6E). In rats, we observed that the growth plate 
still existed until PW28 (the structure of growth plate exists for life in rats), but at this time the growth plate morphology was abnormal, the thickness was obviously thinner (Fig. 6F), which can't continue to promote the growth of limb length.

\section{DISCUSSION}

\section{Dynamic changes of cartilage anlages morphology}

Skeletogenesis initiates at around mid-gestation in the mouse embryo. Limb development begins as mesenchymal cells form condensations from the lateral plate mesoderm. During this process, mesenchymal progenitor cells first move to the future bone sites and then differentiate into chondrocytes to form a cartilage anlage [34]. The cartilage anlage represents the moulds of the future skeletal elements and is gradually replaced by bone during intracartilaginous ossification [8]. When mesenchymal condensations form, several layers of mesenchymal cells at the boundary become fibroblast-like cells and eventually establish the perichondrium, chondrocytes at the centre of individual cartilage anlages undergo hypertrophy. The hypertrophic chondrocyte, simply through its size, is the principal engine of bone growth, and is also a master regulatory cell. Hypertrophic chondrocytes direct the mineralisation of their surrounding matrix, attract blood vessels through the production of vascular endothelial growth factor and other factors, and attract chondroclasts (closely related or identical to osteoclasts, which are cells of the macrophage lineage that digest matrix) [20]. Hypertrophic chondrocytes then undergo apoptotic cell death. Bone-forming cells then invade their lacunae and form POC [30]. This study found that, by GD14, chondrocytes in the centre of the cartilage anlage then stop proliferating, enlarge (hypertrophy). At GD17, the chondrocytes in the centre of the cartilage anlage have gone almost immediately from prehypertrophy to the terminal stage, without undergoing overt hypertrophy. Meanwhile, the joint cavity is formed and the complete model of the developing bone and joint has been formed. The cartilage model of the developing bone then increases in size by both interstitial and appositional growth of the chondrocytes. By GD20, a POC can be clearly distinguished.

Once the POC has formed and early intracartilaginous and intramembranous bone formation has proceeded toward either end of the bone, it is at this stage that the entire cartilaginous region at the developing end of each long bone is referred to as the epiphysis (Fig. 3) [32]. The epiphysis is sometimes referred to as the chondroepiphysis, but use of this term should be restricted to the time prior to formation of the SOC. That part of the epiphysis adjacent to the joint is referred to as the articular cartilage. This merges in indistinguishable fashion histologically with the underlying epiphysis cartilage and only reaches its definitive structure at skeletal maturity when the lowest zone of the articular cartilage calcifies persisting in the adult as the zone of calcified cartilage [32]. The epiphysis growth plate, which is also referred to as the physis or simply the growth plate (Fig. 3), is the cytologically and functionally specialised region of the epiphysis responsible for most of the longitudinal growth of a long bone [32].

While POC formation is an embryonic event, SOC formation occurs shortly after birth at the two ends (epiphysis regions) of rat long bones. At this time, the long bone has three ossification centres, one in the diaphysis and the other two are in each end of the cartilage model. SOC separating permanent articular cartilages from growth plates. When the SOC initiates, the typical epiphysis that refer to the entire developing end of the bone appear at both ends of the cartilage model (Fig. 4). This encompasses 3 regions that are initially cartilage: the articular cartilage, the growth plate, also referred to as the epiphysis growth plate or the physis and the epiphysis cartilage which refers to the cartilage mass between the articular cartilage and the growth plate cartilage [32]. The remaining cartilage between the ossification centres now forms a true "growth plate", driving further longitudinal bone growth, as well as the prospective permanent articular cartilages at each end of the bone [13]. During development, the growth plate is still a transient cartilage tissue. The continuous replacement of this cartilage by trabecular bone and bone marrow leads to its pivotal function as the primary source of longitudinal growth of long bones. In humans, the deposition of cartilage ceases at puberty; the metaphysis fuses with the epiphysis and growth stops. In mouse, longitudinal growth slows dramatically at puberty, but the growth plates do not completely disappear [13]. So the growth plate still exists at PW28 in this study, but it has no function of promoting limb growth.

\section{Morphological changes in the development of articular cartilage}

Articular cartilage is a layer of hyaline cartilage that covers the ends of the bone. It is a highly special- 
ised connective tissue. Within each synovial joint, the articular cartilage is uniquely adapted to bear dynamic compressive loads and shear forces throughout the joint's range of motion [12]. Adult articular cartilage is typically avascular, alymphatic and aneural, it only depends on its unique cellular component - chondrocytes to construct, maintain and remodel extracellular matrix (ECM), with a low cell density and the limited ability of chondrocytes to proliferate [21]. The cells were surrounded by a large number of ECM (type II collagen, proteoglycan). This cartilage can perform continuous functions, such as painless joint activity and elastic recovery.

Most scholars hold the intermediate compartment of the histological interzone gave rise to articular cartilage, while cells in outer interzone layers participate in the initial epiphysis lengthening of long bones [17]. Derived from interzone cells, these periarticular, non-hypertrophic chondrocytes remain as chondrocytes during SOC formation and at the same time develop into articular cartilage with a lubricated surface for smooth joint articulation and load absorption and transmission [11]. Its underlying epiphysis cartilage is completely remodelled into subchondral trabecular bone and the subchondral bone plate. Resorption of the joint interzone leads to formation of the joint cavity and articular cartilage surface. Before the SOC appeared, that part of the epiphysis adjacent to the joint is referred to as the articular cartilage. This merges in indistinguishable fashion histologically with the underlying epiphysis cartilage [18]. SOC form postnatally, separating permanent articular cartilages from transitional growth plate cartilages. The skeletal structure of the limb during development is an ideal model to study the generation of transitional cartilage and permanent cartilage. Articular chondrocytes usually display a stable phenotype and do not undergo the processes characteristic of the growth-plate chondrocytes, such as rapid proliferation followed by cell-cycle exit, hypertrophy and apoptosis [19]. The growth plate is a transient structure and its physiological fate is to be replaced by bone; it is markedly different from articular cartilage that is designed to be permanent, ideally lasting unblemished for the entire lifetime of the individual [18].

Postnatal growth, the articular cartilage undergoes a series of tremendous structural and functional changes [12]. The final structural-maturation change at the undersurface of the articular cartilage, at skeletal maturation, the lowest level of the articular carti- lage becomes calcified and this layer persists through adult life, after formation of the subchondral bone plate, involves calcification of the lowest zone of articular cartilage and appearance of the tidemark that separates the radial zone of the articular cartilage from the calcified zone. In this study, the formation of the tibia and the differentiation of chondrocytes in Wistar rats were firstly observed at GD 14, 17, 20 and PW 2, 6, 28. The results showed that: at GD14, there was no obvious joint space in the limb bud, the whole cartilage anlage is composed of hyaline cartilage, and the articular cartilage can't be recognised in morphology. At GD17, the joint cavity is visible, and the articular cartilage is closely connected to the epiphysis growth plate, but the morphology of articular chondrocytes is indistinguishable from that of the resting area of the epiphysis growth plate. At PW2, cartilage canals appeared in articular cartilage and epiphysis cartilage (Fig. 5), there is no typical layered structure of mature articular cartilage, which can be roughly divided into 3 layers: the surface chondrocytes were flat, the middle layer chondrocytes were large in size and aggregated in groups; the deep layer was adjacent to the SOC, and the chondrocytes were hypertrophic. It is worth mentioning that the chondrocytes in the SOC of epiphysis are lack of proliferative phase, chondrocytes directly differentiate into hypertrophic chondrocytes from resting chondrocytes, without obvious proliferation stage and pre-hypertrophy stage. At PW6, the articular cartilage gradually became thinner, the layers of chondrocytes decreased, the number of hypertrophic chondrocytes decreased, at this time, the articular cartilage appears layered structure, but it still does not have the typical 4 layers structure of mature articular cartilage, the tidemark is not visible and there is no typical subchondral bone morphology. With increasing age, the articular cartilage becomes thinner; at PW12 typical 4-layered structures of articular cartilage can be observed, they are shallow, radioactive, deep and calcified layers, the tidemark between the deep layer and the calcified layer is clearly visible, indicating that the articular cartilage has matured (Fig. 6).

\section{Formation and development of POC}

Our observation showed that, at GD14, at the position where the POC is about to appear in the cartilage anlages, chondrocytes are clustered and distributed in a nest-like manner. The volume is larger than that at both ends, and the intercellular space is enlarged (Fig. 2). With the continuous growth and 
development, the chondrocytes in this area became hypertrophic at GD17. And by GD20, the typical POC structure could be seen. These data suggest that chondrocyte hypertrophy began on GD14, intracartilaginous ossification began on embryo GD15, and POC appeared on GD18-GD19.

Ossification in developing long bones starts in the perichondrium, the connective tissue surrounding the cartilage anlage [13]. At 14 days, chondrocyte hypertrophy occurs in the centre of the developing model of the major long bones, the cellular tissue surrounding the hypertrophic cartilage differentiates into periosteum with an outer fibrous layer and an inner osteoprogenitor-cell layer [13]. At this time, intramembranous periosteal-bone formation begins at the periphery of the middle of the model of the developing bone. The hypertrophic chondrocytes direct adjacent perichondrial cells to become osteoblasts; where cells differentiate into osteoblasts that start to deposit and mineralise a structure called the "bone collar" around the cartilage anlage [20]. This bone collar forms the initiation site of the cortical bone, the dense outer envelope of compact bone that provides most of the strength and rigidity to the long bones. Meanwhile, prehypertrophic chondrocytes in the centre of the cartilage anlage become hypertrophic chondrocytes and finally reach a terminal differentiation stage. Hypertrophic chondrocytes direct the mineralisation of their surrounding matrix, attract blood vessels through the production of vascular endothelial growth factor and other factors, and attract chondroclasts [20]. The actual process of "intracartilaginous ossification" is triggered shortly thereafter, around GD14-GD15 in mouse, when the hypertrophic chondrocytes in the centre of the cartilage anlage become invaded by blood vessels along with osteoclasts and osteoprogenitors from the surrounding perichondrium [13]. As a result, the cartilage anlage is progressively eroded and replaced by trabecular bone and bone marrow, a region initially termed the POC [3]. The ends of the long bones (with the exception of the metatarsals and phalanges) start to show epiphysis shaping (Fig. 3). In the metaphysis, hypertrophic cartilage of the epiphysis growth plate is continually replaced with trabecular bone, a process that relies heavily on angiogenesis and mediates longitudinal bone growth [13]. The intracartilaginous and intramembranous bone formation then extend proximally and distally toward the ends of the bone. The physes at the end of the bone and the surround- ing periosteal intramembranous bone-formation sequence finally reach their farthest relative extent. However, in terms of actual bone-tissue formation, the intramembranous sequence is always slightly in advance of the intracartilaginous sequence, both temporally and spatially. These sequences may be separated by only a few hours.

\section{Formation and development of SOC}

Developing epiphysis consists of articular cartilage, epiphysis cartilage and epiphysis growth plate or physis. The SOC develops within the epiphysis cartilage of developing long bones. The cartilage canal development stage is the initial event preceding the appearance of SOC. When the SOC initiates, several cartilage canals appear at the proximal of the epiphysis cartilage, the epiphysis cartilage is excavated by canals invaginating from the periarticular region. The canals carry nutrients, blood vessels, growth factors and hormones to the centre of the epiphysis cartilage. At this time, chondrocytes at the centre of the epiphysis cartilage undergo hypertrophy and matrix mineralisation. The cartilage canals are oriented toward the position where the SOC was about to form. Finally, the inner ends of the canal fuse and expand by continued excavation of surrounding hypertrophic chondrocytes to create the space for bone and bone marrow, thus establishing the SOC [5]. Once SOC is formed, it separates permanent articular cartilages from transitional growth plate cartilages. It was concluded that the cartilage canals of foetus play great roles in formation of SOC. But with the continuous development and maturity, the cartilage canals in the cartilage eventually disappeared. In this study, we observed that chondrocytes in the central region of epiphysis cartilage began to differentiate and hypertrophy at 7 days after birth. Shortly afterward, several cartilage canals appear at the proximal of the epiphysis cartilage. The chondrocytes around the ends of the cartilage canal are hypertrophy, degeneration, ECM calcification. But the cartilage canal disappeared without being visible at PW3.

When the SOC appears, a centripetal and continuous new bone formation process begins in the epiphysis cartilage, which includes the process of chondrocyte proliferation, hypertrophy, transformation, apoptosis and vascular tissue invasion, with the growth of epiphyses, bone can continue to develop. Generally, the formation of SOC plays an important role in normal joint function. The formation and nor- 
mal development of SOC is the guarantee of normal development of limb morphology and function. The development disorder of ossification centre in different parts may cause different limb deformity, skeletal developmental disorders or joint movement disorder. If the formation or development of cartilage canal is damaged for some reason, the development and growth of epiphysis cartilage and the formation of ossification centre will be seriously damaged, leaving the deformity that is difficult to be corrected, such as absence of femoral head, pathological dislocation of hip, etc. If the epiphysis border between the SOC and the growth plate is not formed, the growth plate lacks a blood supply barrier [29, 33], the infection of epiphysis and metaphysis spreads through the communication tube, which is the anatomical basis for the pathological evolution of acute haematogenous osteomyelitis in infants. Osteochondrosis of the epiphysis is an ischemic disease, and Carlson et al. [7] believe that it is associated with abnormal degeneration of cartilage canals. It is expected that through further research, to reveal its aetiology, so as to put forward targeted treatment measures. Therefore, the cartilage canals in epiphysis cartilage has important significance for the growth and development of cartilage.

Howship first described the vascular channel in cartilage in 1815 and named it "cartilage canals" [15]. As for the formation of cartilage canals, some scholars advocate the theory of "passive inclusion", they think that part of the connective tissue in the perichondrium differentiates into new cartilage, which wrapped the blood vessels and surrounding connective tissue in the original pericardium in cartilage and became the rudiment of the cartilage canals, and it grows and expands as the cartilage develops. Other scholars advocated the theory of "active invasion" and believed that cartilage canals were formed by the dissolution of cartilage matrix and the subsequent active extension of pericarpium vessels and surrounding connective tissue [31]. In this study, we observed that the perichondrium in the epiphysis cartilage in the superficial part of the epiphysis cartilage has an inner depression, or the structure is simple, the deep cartilage tube branches are complex, and the cartilage canals wall is continuous with the perichondrium. These phenomena support the "active invasion" theory of cartilage tube formation. Cartilage canals formation is due to invagination developing at specific sites on the perichondrium. However, the reason why these outbreaks occur in some parts of the perichondrium and not in others remains unknown. The first epiphysis development pattern begins with cartilage canal formation in epiphysis cartilage have proposed three stages of spatial cartilage canal development: superficial, intermediate and deep. Short canals are formed in the superficial stage where the cartilage matrix is not calcified and chondrocytes are not hypertrophied. The canals increase in length during the intermediate stage; the matrix is not mineralised and chondrocytes obtain their level of hypertrophy. The cartilage matrix becomes mineralised during the deep stage.

While POC and SOC formation share many similarities, several key characteristics exist to distinguish these two processes. During POC initiation, invading vessels originate from the perichondrium that has already become the bone collar containing osteoprogenitors and mature osteoblasts. This bone collar eventually expands to become the cortical bone. In contrast, during SOC initiation, invading vessels originate from periarticular region consisting of several layers of flattened fibroblast-like chondrocytes aligned parallel to the surface [34]. While hypertrophic chondrocytes, mineralised ECM, and vessel invasion appear to be involved in both processes, these events are coupled in POC formation but uncoupled in the invagination and cartilage canal stages of SOC formation. As a result, the molecular and cellular mechanisms involved in the early initiation of SOC canal initiation remain unknown [34].

\section{Growth plate and intracartilaginous osteogenesis}

During the formation of $\mathrm{POC}$, the central replacement of hypertrophic chondrocytes with deposition of bone on the calcified cartilage cores encompasses what is referred to as the intracartilaginous mechanism. In the periosteal region intramembranous bone formation extends the periosteal new bone sleeve. The periosteal development is always spatially somewhat more advanced toward either end of the bone than the central endochondral development. As this developmental sequence works its way toward either end of the bone, the cartilage forms itself into a specifically structured region referred to as the epiphysis growth plate (physis) (Fig. 3) [32]. Nilsson et al. [28] divided the epiphysis growth plate into: epiphysis cartilage, resting zone, proliferative zone, proliferation-hypertrophic transition zone and hypertrophic zone by histological characteristics. The chondrocytes in the epiphysis cartilage are round and have no regular orientation. The resting area is 
adjacent to the epiphysis cartilage. The resting zone is populating by undifferentiated chondrocytes serving as a reservoir of stem-like progenitor cells which can rapidly generate proliferating chondrocytes [1]. Next is the proliferation zone which appears as a series of columns aligned parallel to the long axis of the bone. As these cells replicate, the two daughter cells line up parallel to the long axis, to maintain the columnar organization. Type II collagen represents a major component of the cartilage matrix in the resting and proliferative zones [6]. Chondrocytes then exit the cell cycle to become prehypertrophic and switch to produce type $X$ collagen. Prehypertrophic chondrocytes increase in size and become hypertrophic cells that stop expressing type II collagen but continuously express type $X$ collagen [16]. Eventually, hypertrophic chondrocytes reach the terminal stage with matrix mineralisation at the metaphyseal side. At the chondro-osseous junction, the invasion of hypertrophic cartilage by blood vessels, osteoclasts, and osteoprogenitors, together with the terminal differentiation of hypertrophic chondrocytes, leads to degradation and remodelling of the hypertrophic cartilage ECM into trabecular bone [34]. Calcification occurs in the matrix of the lower margins of the hypertrophic zone but involving almost exclusively the longitudinal septae along the long axis of the bone, whereas the transverse septae are either not mineralised or only slightly mineralised. The vascular invasion from below progresses two or three cells deep into the lower margin of the hypertrophic zone and in association with this vascular invasion of the hypertrophic cell regions there is deposition of bone by osteoblasts on the persisting calcified cartilage matrix [32].

After birth, in the long bone, the SOC is successively formed at the end of the cartilage models, the remaining cartilage between the ossification centres now forms a true "growth plate", driving further longitudinal bone growth that is the intracartilaginous ossification, as well as the prospective permanent articular cartilages at each end of the bone. Similar to the epiphysis growth plate, the growth plate is comprised of four discrete zones easily identifiable by histological analyses (Fig. 4). The chondrocytes in the growth plate like the chondrocytes in the epiphysis growth plate undergo stepwise maturation from resting, proliferative, prehypertrophic, and finally hypertrophic and mineralized stages [34]. The growth plate, which increases in width with age, but hardly changes in thickness, is entirely occupied by successive zones leading to intracartilaginous ossification [22]. At the top of the growth plate, round chondrocytes no longer proliferate rapidly and are called resting or reserve chondrocytes, and probably serve as precursors for the flat proliferating columnar chondrocytes [20]. There is a well-defined subchondral bone plate, which is called epiphysis border, on the side of the SOC adjacent to the growth plate, which can prevent the epiphysis vessels from invading the growth plate and thus avoid its premature differentiation and maturation [22]. When the POC and the SOC expand and eventually fuse, the growth plate disappears, which means the maturation of the skeleton. In humans, the growth plate fuses in late puberty, in rodents, this closure can either be greatly delayed or never fully complete [14]. Because of its important role in skeletal growth, any disturbance of signalling pathways required for growth plate development would likely cause skeletal dysplasia. In human, this often presents as a short stature. Mutations in the genes encoding these regulatory molecules result in abnormal chondrocyte behaviour and skeletal dysplasias, most of which are associated with dwarfism, i.e. inadequate growth of the bones that form by intracartilaginous ossification $[25,35]$.

Intracartilaginous ossification is one of the main ways of bone development in mammals, it starts from embryonic period and continues to develop and grow until the end of adolescence. Intracartilaginous ossification is a dynamic process of chondrocytes proliferation and differentiation. The agglutination of mesenchymal cells into the cartilage models is the starting point of intracartilaginous ossification, with the growth and development, mesenchymal cells differentiate into chondrocytes, and chondrocytes further differentiation, hypertrophy, apoptosis and eventually remodelled into bone after vascular invasion [24]. The ECM and angiogenesis stimulants produced by hypertrophic chondrocytes are the key factors to induce vascular invasion and angiogenesis. Neovascularisation transports nutrients to cells involved in ECM absorption and mineral deposition, and provides the necessary signalling factors for the normal development of bone. Studies have shown that osteoclasts and osteoprogenitor cells are always attached to endothelial cells when new blood vessels are formed in the osteogenic zone of the growth plate, and then enter the osteogenic region. Vascular invasion is an essential step in the process 
of intracartilaginous osteogenesis, the invasion of blood vessels provides the necessary nutrients and corresponding cells and factors for the formation of cartilage. Therefore, the invasion of blood vessels is also a sign of cartilage transforming into bone. The proliferation and differentiation of chondrocytes can be orderly carried out in different time and space, which depends on the close regulation of chondrocytes themselves, surrounding matrix, systemic circulation factors, paracrine factors and transcription factors, as well as the precise coordination of activation and inhibition of corresponding pathways. The invasion of blood vessels brings osteoblasts to the cartilage models and forms ossification centre. The expansion and erosion of the ossification centres led to the cartilage anlages eventually developing into bone and permanent articular cartilage.

\section{CONCLUSIONS}

As far as we know, this is the first morphological study to dynamically observe the entire process of cartilage anlages development into articular cartilage and bone through a series of changes from embryo to adult. Our results especially show the formation of POC, the formation of SOC with the participation of cartilage canal and the dynamic process of articular cartilage maturation. The limitation of this study is that only morphological observation was carried out, but no qualitative, quantitative and related molecular mechanisms were studied. Meanwhile, due to the limited funds, continuous observation and comparative analysis of different genders were not carried out. However, the dynamic observation of osteochondral development and the results of morphological characteristics at various important time points in this study is conducive to a deeper understanding of the overall overview of the temporal and spatial regularity of osteochondral development. It is helpful to provide a reference for other research time node selection and morphological analysis.

\section{Funding}

This work was supported by grants from the National Natural Science Foundation of China (Grant No. $81803275,81972036,81673490,81673524)$, the National Key Research and Development Programme of China (grant no. 2017YFC1001300).

Conflict of interest: None declared

\section{REFERENCES}

1. Abad V, Meyers JL, Weise M, et al. The role of the resting zone in growth plate chondrogenesis. Endocrinology. 2002; 143(5): 1851-1857, doi: 10.1210/endo.143.5.8776, indexed in Pubmed: 11956168.

2. Abubakar AA, Noordin MM, Azmi TI, et al. The use of rats and mice as animal models in ex vivo bone growth and development studies. Bone Joint Res. 2016; 5(12): 610-618, doi: 10.1302/2046-3758.512.BJR-2016-0102. R2, indexed in Pubmed: 27965220.

3. Baumgart M, Wiśniewski M, Grzonkowska M, et al. Morphometric study of the primary ossification center of the fibular shaft in the human fetus. Surg Radiol Anat. 2019; 41(3): 297-305, doi: 10.1007/s00276-018-2147-5, indexed in Pubmed: 30542927.

4. Blaker CL, Clarke EC, Little CB. Using mouse models to investigate the pathophysiology, treatment, and prevention of post-traumatic osteoarthritis. J Orthop Res. 2017; 35(3): 424-439, doi: 10.1002/jor.23343, indexed in Pubmed: 27312470.

5. Blumer MJF, Longato $\mathrm{S}$, Fritsch $\mathrm{H}$. Structure, formation and role of cartilage canals in the developing bone. Ann Anat. 2008; 190(4): 305-315, doi: 10.1016/j.aanat.2008.02.004, indexed in Pubmed: 18602255.

6. Burdan F, Szumiło J, Korobowicz A, et al. Morphology and physiology of the epiphyseal growth plate. Folia Histochem Cytobiol. 2009; 47(1): 5-16, doi: 10.2478/ v10042-009-0007-1, indexed in Pubmed: 19419931.

7. Carlson CS, Hilley HD, Meuten DJ. Degeneration of cartilage canal vessels associated with lesions of osteochondrosis in swine. Vet Pathol. 1989; 26(1): 47-54, doi: 10.1177/030098588902600108, indexed in Pubmed: 2913703.

8. Chijimatsu R, Saito T. Mechanisms of synovial joint and articular cartilage development. Cell Mol Life Sci. 2019; 76(20): 3939-3952, doi: 10.1007/s00018-019-03191-5, indexed in Pubmed: 31201464.

9. Cohen MM. The new bone biology: pathologic, molecular, and clinical correlates. Am J Med Genet A. 2006; 140(23): 2646-2706, doi: 10.1002/ajmg.a.31368, indexed in Pubmed: 17103447.

10. Dash S, Trainor PA. The development, patterning and evolution of neural crest cell differentiation into cartilage and bone. Bone. 2020; 137: 115409, doi: 10.1016/j. bone.2020.115409, indexed in Pubmed: 32417535.

11. Decker RS, Um HB, Dyment NA, et al. Genesis and morphogenesis of limb synovial joints and articular cartilage. Matrix Biol. 2014; 39(1): 5-10, doi: 10.1016/j. matbio.2014.08.006, indexed in Pubmed: 25172830.

12. Decker R. Articular cartilage and joint development from embryogenesis to adulthood. Semin Cell Dev Biol. 2017; 62: 50-56, doi: 10.1016/j.semcdb.2016.10.005.

13. Dirckx N, Van Hul M, Maes C. Osteoblast recruitment to sites of bone formation in skeletal development, homeostasis, and regeneration. Birth Defects Res C Embryo Today. 2013; 99(3): 170-191, doi: 10.1002/bdrc.21047, indexed in Pubmed: 24078495.

14. Emons J, Chagin A, Sävendahl L, et al. Mechanisms of growth plate maturation and epiphyseal fusion. Horm Res Paediatr. 2011; 75(6): 383-391, doi: 10.1159/ 000327788. 
15. Howship J. Experiments and observations in order to ascertain the means employed by the animal economy in the formation of bone. Med Chir Trans. 1815; 6: 263-676.5, doi: 10.1177/095952871500600116, indexed in Pubmed: 20895250.

16. Hunziker EB. Mechanism of longitudinal bone growth and its regulation by growth plate chondrocytes. Microsc Res Tech. 1994; 28(6): 505-519, doi: 10.1002/ jemt.1070280606, indexed in Pubmed: 7949396.

17. Ito MM, Kida MY. Morphological and biochemical re-evaluation of the process of cavitation in the rat knee joint: cellular and cell strata alterations in the interzone. J Anat. 2000; 197 (Pt 4): 659-679, doi: 10.1046/j.14697580.2000.19740659.x, indexed in Pubmed: 11197539.

18. Iwamoto $M$, Higuchi $Y$, Enomoto-Iwamoto $M$, et al. The role of ERG (ets related gene) in cartilage development. Osteoarthritis Cartilage. 2001; 9 (Suppl A): S41-S47, doi: 10.1053/joca.2001.0443, indexed in Pubmed: 11680687.

19. Iwamoto $M$, Ohta $Y$, Larmour $C$, et al. Toward regeneration of articular cartilage. Birth Defects Res C Embryo Today. 2013; 99(3): 192-202, doi: 10.1002/bdrc.21042, indexed in Pubmed: 24078496.

20. Kronenberg HM. Developmental regulation of the growth plate. Nature. 2003; 423(6937): 332-336, doi: 10.1038/ nature01657, indexed in Pubmed: 12748651.

21. Las Heras F, Gahunia H, Pritzker K. Articular cartilage development: a molecular perspective. Orthop Clin North Am. 2012; 43(2): 155-171, doi: 10.1016/j.ocl.2012.01.003.

22. Lee ER, Lamplugh L, Davoli MA, et al. Enzymes active in the areas undergoing cartilage resorption during the development of the secondary ossification center in the tibiae of rats ages 0-21 days: I. Two groups of proteinases cleave the core protein of aggrecan. Dev Dyn. 2001; 222(1): 52-70, doi: 10.1002/dvdy.1168, indexed in Pubmed: 11507769.

23. Long F, Chung Ui, Ohba S, et al. Ihh signaling is directly required for the osteoblast lineage in the endochondral skeleton. Development. 2004; 131(6): 1309-1318, doi: 10.1242/dev.01006, indexed in Pubmed: 14973297.

24. Long F, Ornitz DM. Development of the endochondral skeleton. Cold Spring Harb Perspect Biol. 2013; 5(1): a008334, doi: 10.1101/cshperspect.a008334, indexed in Pubmed: 23284041.

25. Macedo MP, Werner $H$, Araujo Júnior E. Fetal skeletal dysplasias: a new way to look at them. Radiol Bras. 2020; 53(2): 112-113, doi: 10.1590/0100-3984.2018.0140, indexed in Pubmed: 32336826.
26. Mackie EJ, Tatarczuch L, Mirams M. The skeleton: a multi-functional complex organ: the growth plate chondrocyte and endochondral ossification. J Endocrinol. 2011; 211(2): 109-121, doi: 10.1530/JOE-11-0048, indexed in Pubmed: 21642379.

27. Ni Q, Lu K, Li J, et al. Role of $\operatorname{TGF} \beta$ signaling in maternal ethanol-induced fetal articular cartilage dysplasia and adult onset of osteoarthritis in male rats. Toxicol Sci. 2018; 164(1): 179-190, doi: 10.1093/toxsci/kfy080, indexed in Pubmed: 29617878.

28. Nilsson O, Weise M, Landman EBM, et al. Evidence that estrogen hastens epiphyseal fusion and cessation of longitudinal bone growth by irreversibly depleting the number of resting zone progenitor cells in female rabbits. Endocrinology. 2014; 155(8): 2892-2899, doi: 10.1210/ en.2013-2175, indexed in Pubmed: 24708243.

29. Parvaresh KC, Upasani VV, Bomar JD, et al. Secondary ossification center appearance and closure in the pelvis and proximal femur. J Pediatr Orthop. 2018; 38(8): 418-423, doi: $10.1097 / \mathrm{BPO} .0000000000000836$, indexed in Pubmed: 27442214

30. Pazzaglia UE, Congiu T, Sibilia V, et al. Relationship between the chondrocyte maturation cycle and the endochondral ossification in the diaphyseal and epiphyseal ossification centers. J Morphol. 2016; 277(9): 1187-1198, doi: 10.1002/jmor.20568, indexed in Pubmed: 27312928.

31. Peinado Cortés LM, Vanegas Acosta JC, Garzón Alvarado DA. A mechanobiological model of epiphysis structures formation. J Theor Biol. 2011; 287: 13-25, doi: 10.1016/j. jtbi.2011.07.011, indexed in Pubmed: 21810429.

32. Shapiro F. Developmental bone biology. 2001: 3-128, doi: 10.1016/B978-012638651-6/ 50002-2.

33. Tong W, Tower RJ, Chen C, et al. Periarticular mesenchymal progenitors initiate and contribute to secondary ossification center formation during mouse long bone development. Stem Cells. 2019; 37(5): 677-689, doi: 10.1002/ stem.2975, indexed in Pubmed: 30681752.

34. Tower R, Qin L. Chondrocyte cell fate analysis. Encyclopedia of Bone Biology. 2020: 621-631, doi: 10.1016/b978-0-12801238-3.11190-0.

35. Wrmn ML, Cormier-Daire V, Hall C. Nosology nd clssifiction of genetic skeletl disorders: 2010 revision. Am J Med Genet A. 2011; 155A(5): 943-968, doi: 10.1002/ ajmg.a.33909.

36. Yang L, Tsang KY, Tang HC, et al. Hypertrophic chondrocytes can become osteoblasts and osteocytes in endochondral bone formation. Proc Natl Acad Sci U S A. 2014; 111(33): 12097-12102, doi: 10.1073/pnas.1302703111, indexed in Pubmed: 25092332. 